\title{
IAC-19-A6.2.6: NewSpace and its Implications for Space Debris Models
}

\author{
Mr. Samuel Diserens ${ }^{a *}$, Prof. Hugh G. Lewis ${ }^{b}$, Prof. Jörg Fliege ${ }^{c}$ \\ ${ }^{a}$ Department of Aeronautical and Astronautical Engineering, University of Southampton, Southampton SO17 1BJ, \\ UK, S.D.Diserens@soton.ac.uk \\ ${ }^{\mathrm{b}}$ Department of Aeronautical and Astronautical Engineering, University of Southampton, Southampton SO17 1BJ, \\ UK, H.G.Lewis@soton.ac.uk \\ c Department of Mathematical Sciences, University of Southampton, Southampton SO17 1BJ, UK, \\ J.Fliege@soton.ac.uk \\ * Corresponding Author
}

\begin{abstract}
Until two decades ago, the dominance of the space industry by national governments shaped the key characteristics of the spacecraft population and hence the space debris models used to anticipate future orbital populations. The rise of 'NewSpace' with the growth of private sector involvement has brought innovations, disrupting the status quo and changing to the physical characteristics and mission orbits of commercial spacecraft. Analysis of shifts in mass and launch traffic for spacecraft launched between 1980 and 2019 (source: ESA's DISCOS database) suggests significant impacts for debris modelling. Results emphasised the ongoing change towards a more commercially focused space sector. 742 spacecraft were launched in the 1980s, of which 34 were labelled as commercial. By contrast, there were 1292 commercial missions out of 2325 launched in the 2010s - an increase from 4.6\% to $55.6 \%$. These increases correlate with a rise in the number of different organisations operating spacecraft and a clear trend can be seen towards smaller, lower mass spacecraft. This is likely to alter the distributions of fragments generated in collisions compared with the distributions obtained from empirical methods, such as the NASA Standard Breakup model, derived from the fragmentation of larger spacecraft in the 600-1,000 kg range. A study of observed breakup events indicates that the NASA Standard Breakup Model over-estimates the number of large debris released during fragmentations due to collisions or explosion of satellites while also under-estimating the number of small debris. It is believed that this will have a significant impact on the outcomes of simulations of the future debris environment where these fragmentation modes are expected to dominate the generation of new debris over the propulsion based explosions which have been historically prevalent.
\end{abstract}

Keywords: Orbital Debris, Debris Models, New Space, Fragmentation, Breakup

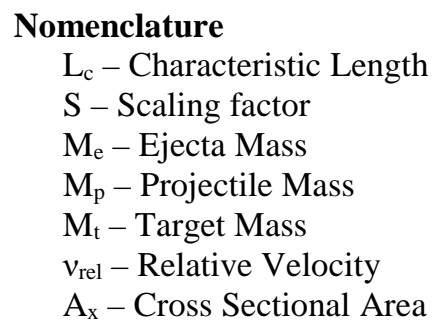

\section{Acronyms/Abbreviations}

Area to Mass Ratio (AMR)

Low Earth Orbit (LEO)

Geosynchronous Earth Orbit (GEO)

Active Debris Removal (ADR)

Radar Cross-Section (RCS)

Polar Satellite Launch Vehicle (PSLV)

\section{Introduction}

The presence of space debris is a well-recognised risk to the safe operation of spacecraft and the ongoing use of the space environment. As of January 2019 there were an estimated 34,000 space debris objects greater than $10 \mathrm{~cm}$ in diameter with a further 900,000 objects greater than $1 \mathrm{~cm}$ with the potential to cause mission-ending damage to spacecraft [1].

Studies of the dynamics of this environment by Kessler and Cour-Palais [2], and by Kessler and Anz-Meador [3] theorised that for certain regions the space debris population may reach critical object densities above which random 
collisions would result in instability leading to self-sustaining growth of the debris population even without additional launches, either to a new equilibrium or exponentially. Numerous computational models $[4,5,6,7,8,9,10]$ have been developed to simulate the space debris environment and to better understand the growth of the debris population over time.

In recent years the space industry has been undergoing a period of expansion and change with a growing start-up culture, often dubbed 'NewSpace' or 'the new space age' [11,12]. This development is generally associated with the increasing involvement of the private investment and changes in priorities compared to traditional government and military-industrial complex operators. Bryce Space and Technology reports a greater than twelve-fold increase in the average number of space investors per year since the year 2000 [13].

The competitive nature of the commercial ecosystem has driven development in different spacecraft technologies and their missions, including the increase in smaller, lighter satellites, novel methods like additive manufacturing, and mass launch events such as PSLV-C37 [14,15,16].

With the current rate of change it is impossible to know what the future of the space industry will look like and how many of these new ventures will survive to maturity [17]. It is acknowledged however, that these changes bring new challenges to be explored and it is expected that there will be implications for the future of the space debris environment [18].

Of particular interest to the authors of this paper is the consequences of these changes not only for the results generated by the current generation of debris models, but for the validity of debris models when applied to this changing environment.

\section{Studying NewSpace trends}

To investigate the trends being introduced by NewSpace, ESA's DISCOS database [19] was examined for information on the key characteristics of spacecraft launched between 1980 and 2019. To provide a means for comparison each of the spacecraft in the database was classified as either military, civil, academic, or commercial based on the nature of the organisation which operated the spacecraft.

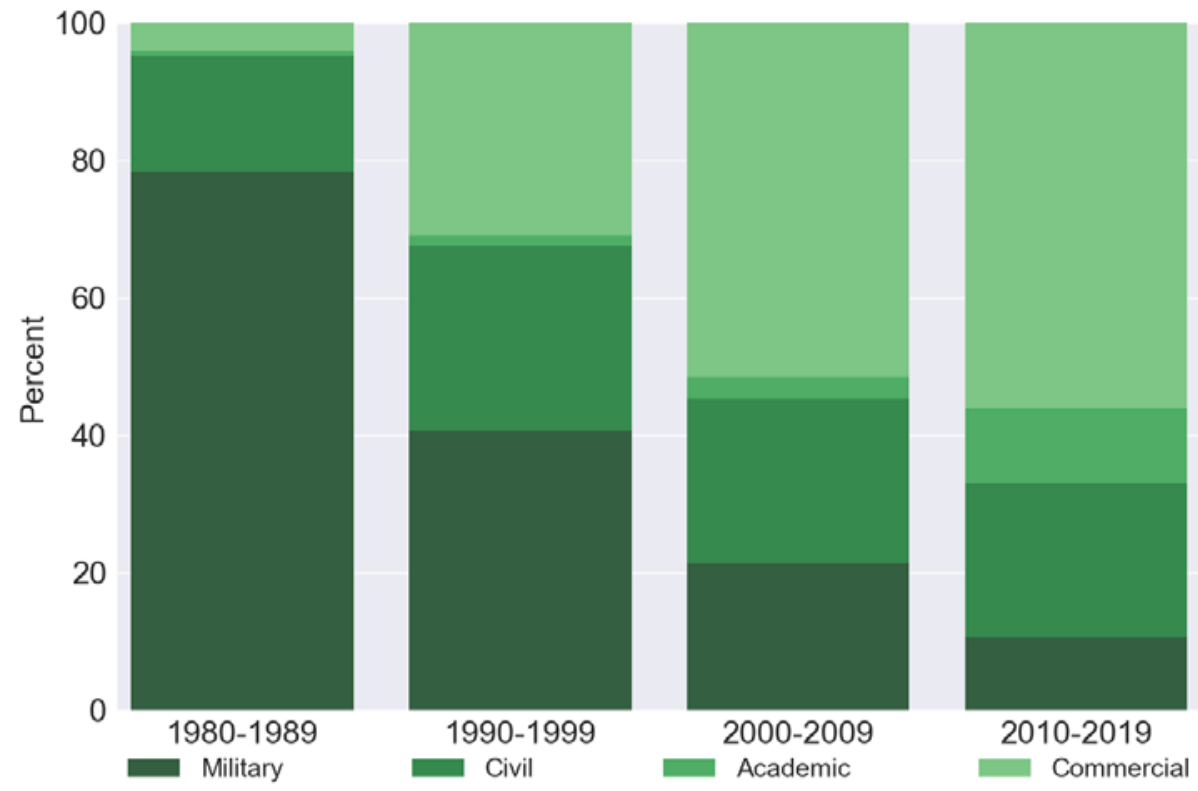

Fig. 1: Percentage of spacecraft launched per decade by organisation type.

A comparison of the prevalence of these different classifications in each decade can be seen in Fig. 1 . The proportion of commercial spacecraft has grown from $4.6 \%$ of the launch population in the 1980 s to $55.6 \%$ in the last decade. At the same time the military share has shrunk from $76.5 \%$ to only $10.5 \%$ of launched spacecraft. This can be attributed in part to the end of the Cold War following the dissolution of the USSR in the early 1990s. However, the absolute numbers of spacecraft launched in each category, shown in Fig.2 below, indicate that this trend is not simply due to comparable spacecraft moving from control of a military or civil organisation to a commercial one. 
The total number of launches in the civil and military categories has been consistently around 700 per decade, while there has been a $213 \%$ increase in the total number of launches from the 1980 s to the 2010 s. Of this increase $208 \%$ is accounted for by increased numbers of commercial and academic spacecraft, from 34 to 1292 and 5 to 253 respectively.

Fig. 3 to Fig. 8 show the results of analysis of trends in the physical characteristics of the spacecraft, notably the mass, size and area to mass ratio (AMR) for commercial spacecraft in each of the Low Earth Orbit (LEO) and Geosynchronous Earth Orbit (GEO) regions using data from the ESA DISCOS database.

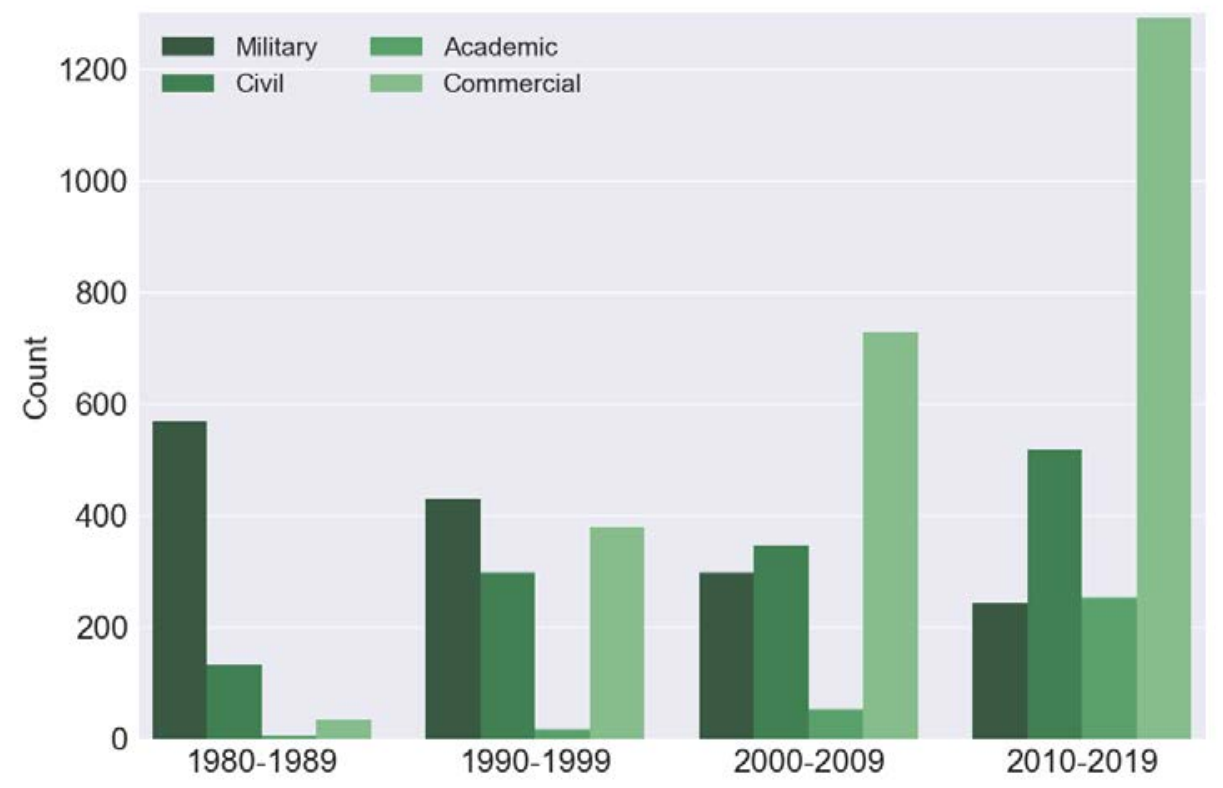

Fig. 2: Count of spacecraft launched per decade by organisation type.

A set of filters was applied to these data before analysis. First the spacecraft type was filtered to remove all rocket bodies, leaving only the payload satellites. Next the results were filtered on mass to remove all spacecraft greater than $10,000 \mathrm{~kg}$. This had the effect of removing all space stations as well as Space Shuttle and other re-supply missions which were classified as outliers due to being either one-off or short duration missions.

The distribution of commercial spacecraft by mass has changed between successive 5 year periods in each of the LEO and GEO regions (as shown in the box and whisker plots in Fig. 3 and Fig. 4). Similarly the distribution in average cross-section (Fig. 5 and Fig. 6) and AMR (Fig. 7 and Fig. 8) have changed for commercial spacecraft over the same periods for the LEO and GEO regions. In these plots the boxes describe the values of the upper and lower quartiles with a central line at the median value while the whiskers extending beyond the box show the distribution of the remaining data. This excludes outliers which are plotted as distinct points and classified as data points falling more than 1.5 times the interquartile range outside the first and third quartiles.

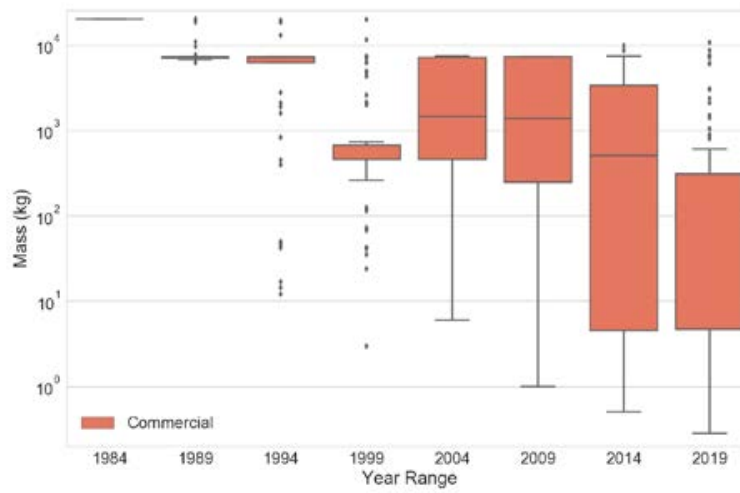

Fig. 3: Variation in mass of LEO spacecraft over time.

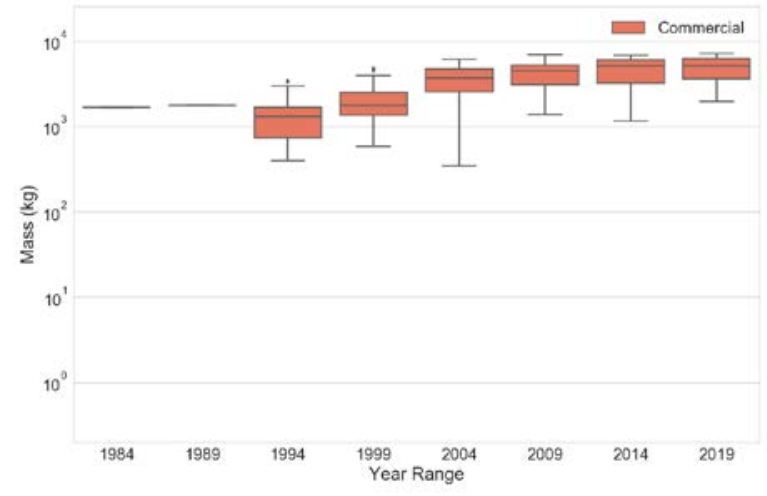

Fig. 4: Variation in mass of GEO spacecraft over time. 


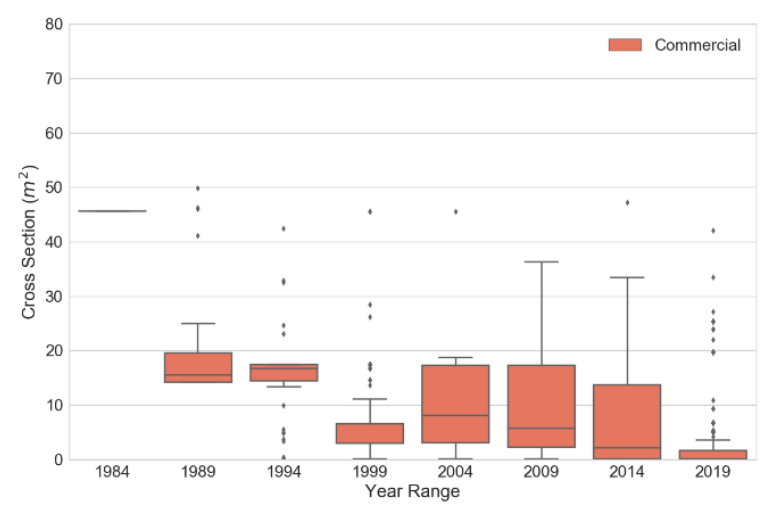

Fig. 5: Variation in cross-section of LEO spacecraft over time.

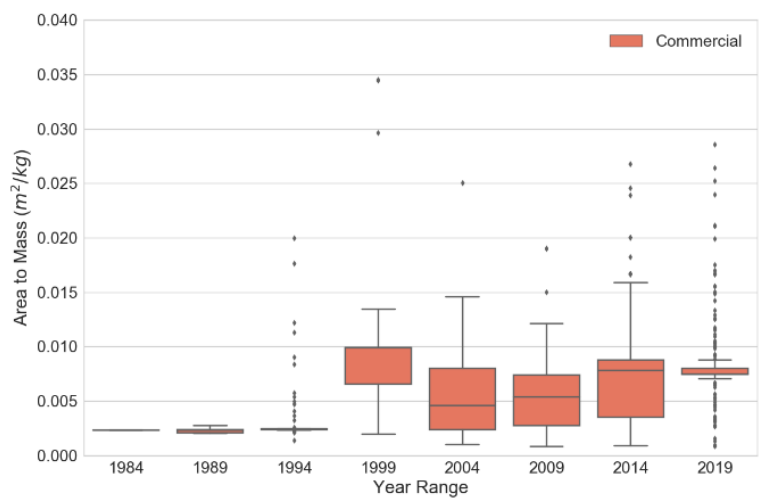

Fig. 7: Variation in area-to-mass ratio of LEO spacecraft over time.

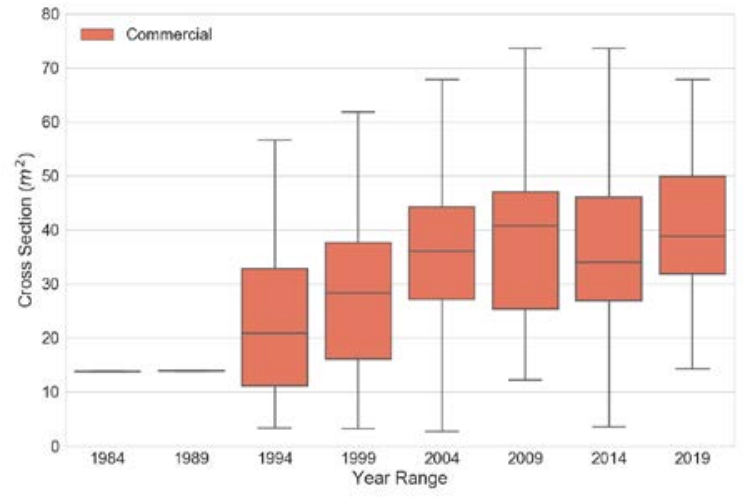

Fig. 6: Variation in cross-section of GEO spacecraft over time.

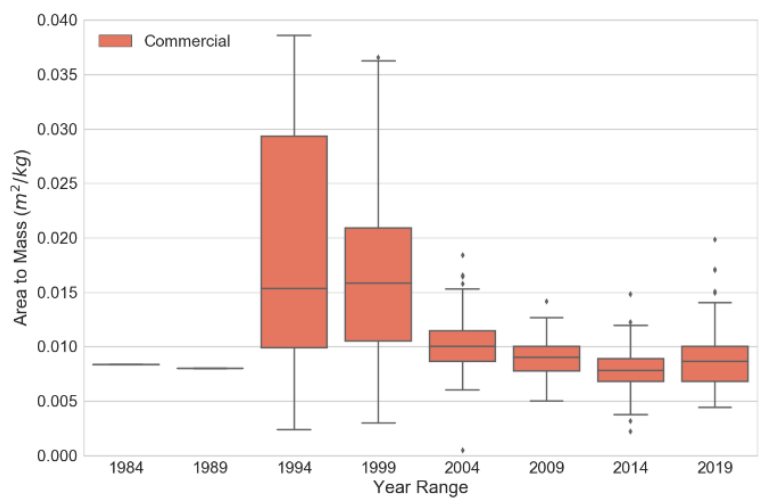

Fig. 8: Variation in area-to-mass ratio of GEO spacecraft over time

The first figure shows a trend to decreasing average spacecraft mass in the LEO region which is particularly significant within the last 5 years with the average mass falling from multiple tons to $500 \mathrm{~kg}$ to only $5 \mathrm{~kg}$. This drop is accompanied by a widening of the distribution to cover a broader range of masses. Over the same period there has been an increasing trend in the GEO region with average mass growing from a minimum of $1300 \mathrm{~kg}$ to $5080 \mathrm{~kg}$.

The next two figures show trends in spacecraft cross-section towards smaller spacecraft in LEO and larger spacecraft in GEO. With average LEO cross section dropping from $15 \mathrm{~m}^{2}$ in the late 1980s and early 1990s to $2 \mathrm{~m}^{2}$ and then to $0.02 \mathrm{~m}^{2}$ and GEO cross section growing from $15-20 \mathrm{~m}^{2}$ to $40 \mathrm{~m}^{2}$, corresponding to the trends in spacecraft mass. However, the results show a greater variation in the average cross-section of GEO spacecraft than there is for LEO spacecraft with a range of $54 \mathrm{~m}^{2}\left(14\right.$ to $\left.68 \mathrm{~m}^{2}\right)$ compared to $3.509 \mathrm{~m}^{2}\left(0.025\right.$ to $\left.3.534 \mathrm{~m}^{2}\right)$. What appears to be the opposite in the plots of spacecraft mass is due to the logarithmic scaling, with GEO spacecraft falling between 1954 $\mathrm{kg}$ and $7075 \mathrm{~kg}$ compared with $4.2 \mathrm{~kg}$ and $1037 \mathrm{~kg}$ for LEO.

This has resulted in much small changes in the AMRs of the spacecraft. Conversely to the other characteristics a convergence of the LEO and GEO AMRs can be seen. For LEO spacecraft the mean value for the AMR has more than tripled from 0.0023 to $0.0074 \mathrm{~m}^{2} / \mathrm{kg}$, while for GEO spacecraft average AMR dropped from $0.015 \mathrm{~m}^{2} / \mathrm{kg}$ in the 1990s to $0.0086 \mathrm{~m}^{2} / \mathrm{kg}$.

One of the reasons behind these trends is the growing popularity of small satellites, such as CubeSats which allow for the easy, standardised construction of spacecraft, often using cheap off the shelf components. The trend to smaller spacecraft has been echoed in the launch industry with several small rockets such as Orbex's Prime, RocketLab's Electron and VirginOrbit's air-launched LauncherOne being brought to market. Additional design changes such as drag sails or the large flat shape of SpaceX's Starlink satellites specifically increase the AMR in order to increase the rate of decay due to atmospheric drag, shortening disposal at end of life.

As well as these innovations in the construction of spacecraft, novel mission types are being explored as companies seek out new markets such as on-orbit servicing, end-of-life management and Active Debris Removal (ADR). Recent years have seen changes in operational characteristics such as the growing trend towards ridesharing, and the launch 
of multiple spacecraft on the same rocket into similar orbits. One result of this practice is that the orbital elements of the spacecraft are clustered into specific regions of altitude and inclination rather than being evenly distributed. At the same time the number of distinct operators has increased, with 167 different organisations launching spacecraft in 2017 with an average of 102 different organisations each year for the last decade, compared to only 18 in the 1980s.

The practice of multi-spacecraft launches is exemplified by large constellations such as Starlink, the first deployment of which included 60 spacecraft on a single Falcon 9 [20]. The orbital clustering will be further exacerbated by the launch cadence required to populate a large constellation like Starlink with SpaceX alone planning on launching up 24 missions, another 1440 spacecraft, into similar orbits in 2020.

\section{NewSpace and Debris Modelling}

There are several key elements to the evolution of space debris and these play different roles in the evolution of the overall debris environment. Component models are used to describe the processes of these elements when simulating the environment (shown in Table 1).

The application of each of these component models is dependent on the different characteristics of individual objects and the environment as a whole. For example, the mass of an object when modelling its fragmentation or its AMR when calculating atmospheric drag. As a result many of these models rely on assumptions about how these characteristics affect the outcome. The previously identified trends in these characteristics since the original development of these models and their assumptions may render them inaccurate for the modern spacecraft population.

Table 1. Key components of debris models

\begin{tabular}{lll}
\hline Process & Role & Example Model \\
\hline Launch & Source & Historic data \\
Fragmentation & Source & Evolve 4.0* [21] \\
Explosions & Sink & Historic data \\
Collision & Sink & Cube Approach [22] \\
& & Orbit-Trace [9] \\
Atmospheric Decay & Sink & Orbital Propagators \\
\hline \hline Orbital Propagation & SGP4 [23] \\
\hline \multicolumn{1}{c}{ Atmospheric Density } & \\
\multicolumn{2}{l}{ Solar Radiation Pressure } & \\
\hline \multicolumn{2}{l}{ Gravitational Influence (Earth/Moon/Sun) } & \\
\hline
\end{tabular}

* the NASA Standard Breakup Model

The process of fragmentation has been identified as one of the most important areas for consideration, with breakups due to collisions in particular being expected to become the dominant source of new debris in the future environment [2,3]. In the wider context of modelling the debris environment, models of how objects undergo fragmentation are important for identifying not only how many new objects are added to the environment but also their key characteristics, e.g. size, mass and delta-v relative to the parent body.

Of the current generation of debris models almost all [24] make use of the EVOLVE 4.0 NASA Standard Breakup Model making it of particular interest for further investigation. The only notable exception to this is Aerospace Corporation's ADEPT model which uses an in house tool known as IMPACT [25]. In the NASA model, first described in 2000, debris fragments are generated using the size, or characteristic length $\left(L_{c}\right)$, as the independent variable which is then used to generate the mass, AMR, and ejection velocity. The distribution of fragments according to $\mathrm{L}_{\mathrm{c}}$ is defined as follows:

$$
N\left(L_{C}\right)=\left\{\begin{aligned}
6 \mathrm{~S} \mathrm{~L}_{\mathrm{C}}^{-1.6}, & \text { if Explosion } \\
0.1 M_{e}^{0.75} L_{C}^{-1.71}, & \text { if Collision }
\end{aligned}\right.
$$

The power law equation for explosions was developed based on observations of fragments from the explosions of several different rocket bodies and is expected to be valid for the explosion of upper stages with masses between 600 $\mathrm{kg}$ and $1,000 \mathrm{~kg}$. For explosions due to other means, such as battery explosions, or of other types of spacecraft a value between 0.1 and 1 is used for the type-dependant scaling factor, $\mathrm{S}$. The 600 to $1,000 \mathrm{~kg}$ mass range is very specific and is no longer consistent with average masses for upper stages or the spacecraft masses analysed in Fig. 3 and Fig. 4 which show that most spacecraft launched in recent years have been below or above this mass range for the LEO and GEO regions respectively.

For the collision scenario the ejected mass, $\mathrm{M}_{\mathrm{e}}$, is defined by equation (2) where $\mathrm{M}_{t}$ and $\mathrm{M}_{\mathrm{p}}$ are the masses of the target and projectile objects respectively, and $v_{\text {rel }}$ is the relative velocity between the two objects at collision: 


$$
M_{e}=\left\{\begin{aligned}
M_{t}+M_{p}, & \text { if Catastrophic } \\
M_{p}\left(\frac{v_{r e l}}{1000}\right)^{2}, & \text { if Non - catastrophic }
\end{aligned}\right.
$$

A catastrophic collision is defined as one where the kinetic energy of the impactor is greater than 40 joules per gram of target mass. Due to a lack of on-orbit examples the equation governing collisions was developed using ground based hypervelocity test such as the SOCIT test series and the Solwind P-78 anti-satellite test. The basic implementation of this model makes the assumption that all collisions which take place will involve the entire body of each object. This does not take into account spacecraft consisting of multiple connected structures. For instance the presence of appendages such as gravity gradient booms, or deployed solar panels.

Historically debris generation has been dominated by fragmentation of spacecraft; accounting for $65 \%$ of growth in the tracked object catalogue since 2008 and 53\% of the total catalogue according to the $15^{\text {th }}$ edition of NASA's 'History of On-Orbit Satellite Fragmentations' [26]. Of the 242 satellites believed to have broken up since 1957, accidental collisions between objects only account for $2.5 \%$ of events compared to the number of propulsion-based explosions (44.2\% of events). However, while the absolute number of accidental collisions is small they account for a much greater proportion of catalogued debris (11.6\%). This suggests that the collision events, when they occur, are far more significant to the overall evolution of the debris environment.

Mitigation measures to avoid the release of debris, such as developments in spacecraft design and through procedures such as passivation at end-of-life, have been implemented to reduce the probability of explosion for individual spacecraft. The rate at which fragmentations have occurred has remained consistent at about 4-5 breakups per year since 2000 despite an exponential growth in the number of spacecraft on orbit. It indicates that mitigation efforts are somewhat successful. One side-effect of this is expected to be that propulsion based explosions may become less prevalent in the future.

It is expected that, while current trends indicate that the rate of explosion events will remain constant, the rate of collisions will increase. NewSpace will continue to exacerbate the issue as more spacecraft are launched and operated by an ever wider range of organisations.

\section{Testing the NASA Standard Breakup Model}

An investigation was undertaken into the results of the NASA Standard Breakup Model for scenarios representative of future breakups. In order to achieve this an implementation of the breakup model was used to simulate several historic fragmentation events. The results of the simulations were then compared against observations of the debris fragments produced by each event.

Detailed information on the number and size of fragments released from each parent object was extracted from the Space Track catalogue (https://www.space-track.org/). Within the catalogue, fragments are categorised as being either 'Small', 'Medium', or 'Large' based on their observed radar cross-section (RCS):

$$
\left\{\begin{array}{rlrl}
R C S<0.1 \mathrm{~m}^{2}, & & \text { Small } \\
0.1 \mathrm{~m}^{2}<R C S<1.0 \mathrm{~m}^{2}, & & \text { Medium } \\
1.0 \mathrm{~m}^{2}<R C S \quad, & \text { Large }
\end{array}\right.
$$

Comparative values for the characteristic length were calculated using the relation defined in the original paper on the NASA Standard Breakup Model:

$$
A_{x}=0.556945 L_{C}^{2.0047077}
$$

This resulted in the following size categories:

$$
\left\{\begin{aligned}
0.1 m<L_{C}<0.425 m, & \text { Small } \\
0.425 m<L_{C}<1.339 m, & \text { Medium } \\
1.339 m<L_{C}, & \text { Large }
\end{aligned}\right.
$$

A minimum characteristic length of $0.1 \mathrm{~m}$ was assumed for the small fragment category. This was chosen based on the stated tracking limits of the Space Surveillance Network which USSTRATCOM uses to populate the Space-Track database. Combining this with the number of fragments in each size category then gave the cumulative number of fragments greater than three different characteristic lengths for each of the scenarios.

An analysis of the individual RCS values of the observed fragments would be preferable to improve comparability of the results by allowing comparison against a larger number of bins. However, at present these data are not readily available in the public domain. 
A range of different fragmentation events were selected from the sub-categories of: upper-stage explosions; satellite explosions; and catastrophic collisions. The decision was taken to separate the explosion events between satellites and upper-stages based on the hypothesis that different results would be seen due to differences in the design and materials used as well as the cause of the explosion.

The first of these categories, the upper stage explosions, is examined in Table 2 and Fig. 9. Four different breakup scenarios were simulated for objects with a range of masses. The first three cases were traditional upper-stages similar to those observed in the original development of the model. These cases provide a reference point for the performance of the model for the traditional spacecraft population. The fourth scenario, the explosion of a Pegasus manoeuvring stage, involved an air-launched rocket body with a much lower mass, similar to the small launchers becoming more common with the advent of NewSpace, and in particular to Virgin Orbit’s LauncherOne.

The NASA Standard Breakup Model was used to simulate each scenario in Table 2 and the resulting fragment size distribution was plotted in Fig. 9 along with the cumulative number of fragments observed for each of the three size categories. By fitting a line to the observed data points a value was calculated for the exponent of a power law for comparison with the value of -1.6 used in equation 1.

Table 2. Observed examples of explosions of upper stage rocket bodies.

\begin{tabular}{|c|c|c|c|c|c|}
\hline \multirow[t]{2}{*}{ Parent Body } & \multirow[t]{2}{*}{ Mass (kg) } & \multirow[t]{2}{*}{ Length (m) } & \multicolumn{3}{|c|}{ Number of Fragments } \\
\hline & & & Large & Medium & Small \\
\hline Long March 4 third-stage & 1000 & 5 & 7 & 64 & 372 \\
\hline Ariane 1 third-stage & 1400 & 10 & 7 & 161 & 499 \\
\hline PSLV* final-stage & 900 & 3 & 2 & 30 & 372 \\
\hline Pegasus maneuvering stage & 97 & 1 & 0 & 72 & 755 \\
\hline
\end{tabular}

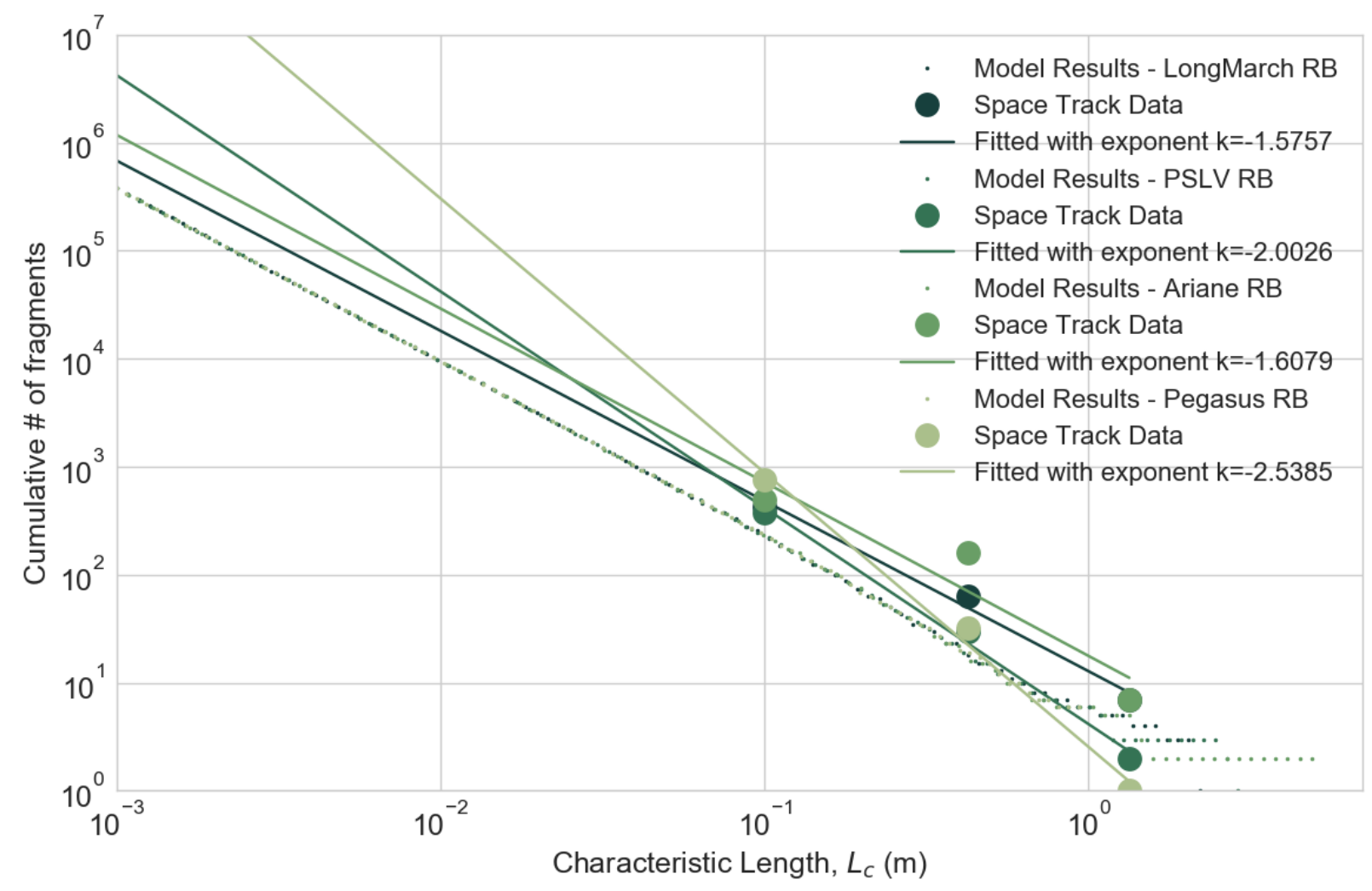

Fig. 9: Comparison of fragment size distribution of observed data against model results for rocket body explosions.

The results of this line fitting for the LongMarch and Ariane rocket bodies appear to be consistent with the model, with fitted values of -1.5757 and -1.6079 respectively. However, the slightly lower mass Polar Satellite Launch Vehicle (PSLV) rocket body deviated from this value with a fitted exponent of -2.0026 and the significantly smaller, $97 \mathrm{~kg}$ Pegasus rocket body deviates noticeably with a value of -2.5385 . 
This suggests that, while the model performs well for traditional upper-stages around the 600-1000 kg range, there is an unaccounted for relationship between the size distribution of fragments and the initial mass of the fragmenting spacecraft. The result of this relationship is that the number of large fragments produced by the breakup of small upper stages is being over predicted by the NASA Standard Breakup Model. It is noted that applying a scaling factor to these results would correct either the number of large fragments predicted or the number of small fragments, but not both.

Table 3 and Fig. 10 address the category of satellite explosions using relatively recent confirmed satellite breakups as scenarios. One of these was the breakup of the Russian Cosmos-2421 while the other two breakup events were of US earth observation satellites, NASA's NOAA-16 and the U.S. Air Force's DMSP-F13. The cause of the Cosmos2421 breakup remains uncertain but an explosion is assumed due to the number of fragments generated. The two US satellites are believed to have fragmented as a result of battery explosion due to overcharged battery packs.

The masses of these spacecraft is more representative of traditional spacecraft than of the NewSpace trends observed. However, the different explosion type has the potential to be a more significant proportion of future explosions due to increasing levels of passivation. This makes the scenarios interesting as an alternative to the historic explosions of propellant tanks.

Table 3. Observed examples of explosions of satellites.

\begin{tabular}{lrrrrr}
\hline Parent Body & Mass (kg) & Length (m) & \multicolumn{3}{c}{ Number of Fragments } \\
\cline { 3 - 6 } & & & Large & Medium & \multicolumn{1}{c}{ Small } \\
\hline NOAA-16 & 1403 & 1 & 1 & 3 & 455 \\
DMSP-F13 & 815.66 & 3.7 & 1 & 1 & 238 \\
Cosmos-2421 & 3150 & 2 & 1 & 10 & 498 \\
\hline
\end{tabular}

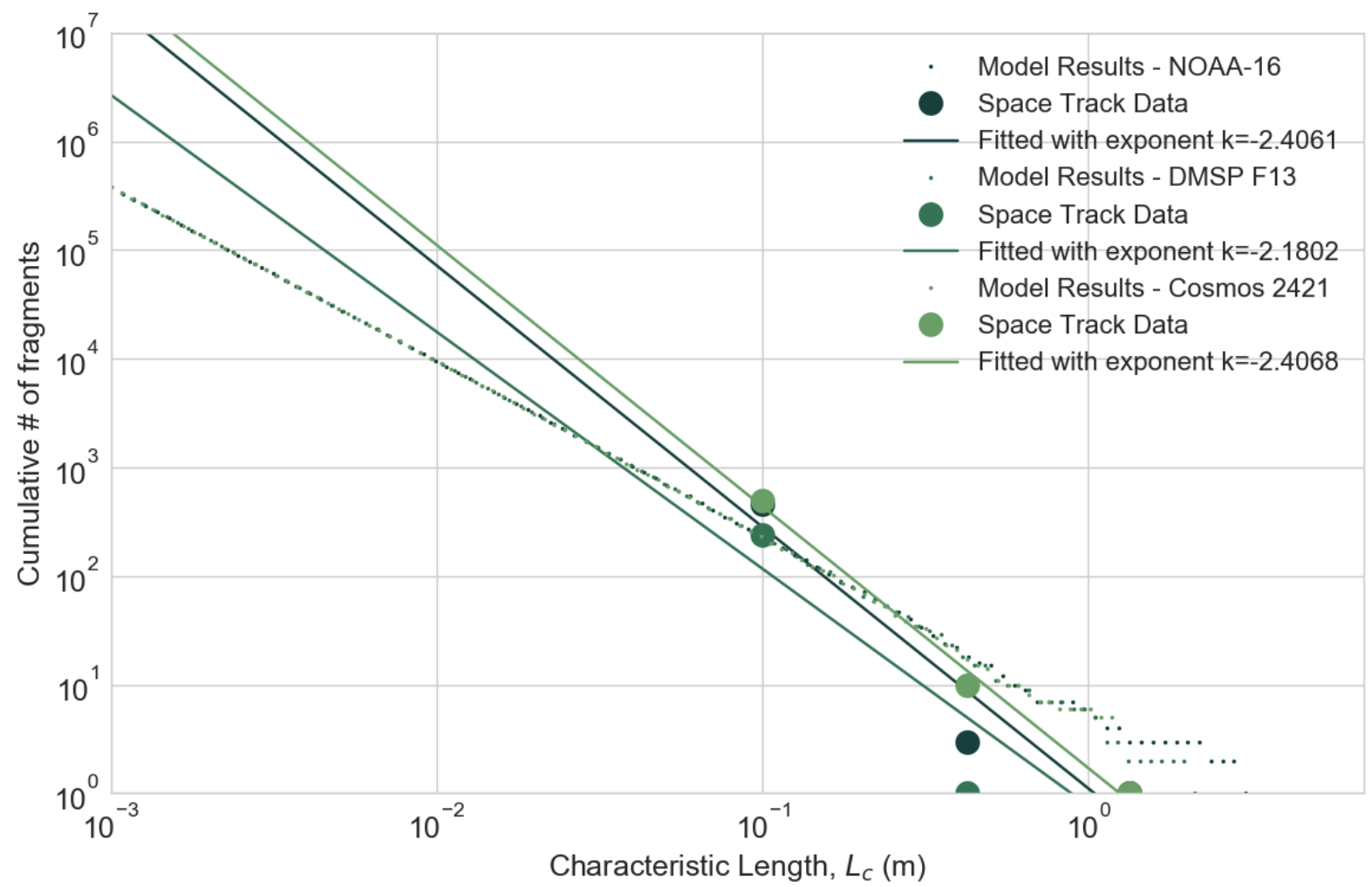

Fig. 10: Comparison of fragment size distribution of observed data against model results for satellite explosions.

As with the rocket body explosions the results of the NASA Standard Breakup Model were plotted against the numbers of observed fragments and a line fit was used to compare against equation 1. Values of $-2.4061,-2.1802$ and -2.4068 were calculated for NOAA-16, DMSP-F13 and Cosmos-2421 respectively. These fitted trend lines suggest that the model may currently be over-predicting the number of large fragments generated with length greater than 30 $\mathrm{cm}$, and under-predicting the number of small fragments with lengths less than $5 \mathrm{~cm}$. These results indicate that the breakup model is less suitable for simulating the breakup of satellites, either due to differences in structure or due to 
the source of the explosion. In contrast to the previous results there appears to be a weaker relationship between the mass of the spacecraft and the slope of the fitted line, it does, however, suggest that alternative parameters might be needed when modelling the breakup of satellites.

The final category of spacecraft collisions is explored in Table 4 and Fig. 11. While the masses of these spacecraft were not consistent with the NewSpace trends identified these events were the first catastrophic collision events to occur since the development of the model and so provide insight into the performance of the model in a future collision dominated environment.

The results are consistent with the results for satellite explosions, with the number of observed fragments for the size groupings being of a similar scale to the simulation results. The fit of the data to the modelled relationship between size and number of fragments, however, again appears to deviate, with more fragments observed than predicted for the small size band, and fewer for the large size band.

Table 4. Observed examples of collisions of spacecraft.

\begin{tabular}{|c|c|c|c|c|c|c|c|}
\hline \multirow[t]{2}{*}{ Parent Body } & \multirow{2}{*}{$\begin{array}{l}\text { Mass } \\
\text { (kg) }\end{array}$} & \multirow{2}{*}{$\begin{array}{l}\text { Impact } \\
\text { Mass (kg) }\end{array}$} & \multirow{2}{*}{$\begin{array}{l}\text { Relative } \\
\text { Velocity(m/s) }\end{array}$} & \multirow[t]{2}{*}{ Length (m) } & \multicolumn{3}{|c|}{ Number of Fragments } \\
\hline & & & & & Large & Medium & Small \\
\hline Cosmos-2251 & 900 & 556 & 11,570 & 2 & 1 & 29 & 1668 \\
\hline Iridium-33 & 556 & 900 & 11,570 & 4 & 1 & 24 & 628 \\
\hline Fengyun-1c* & 850 & 600 & 9,000 & 2 & 0 & 34 & 3408 \\
\hline
\end{tabular}

* Some uncertainty exists on the exact characteristics of the Fengyun-1C collision as details of the kinetic kill vehicle used remain classified by the Chinese administration.

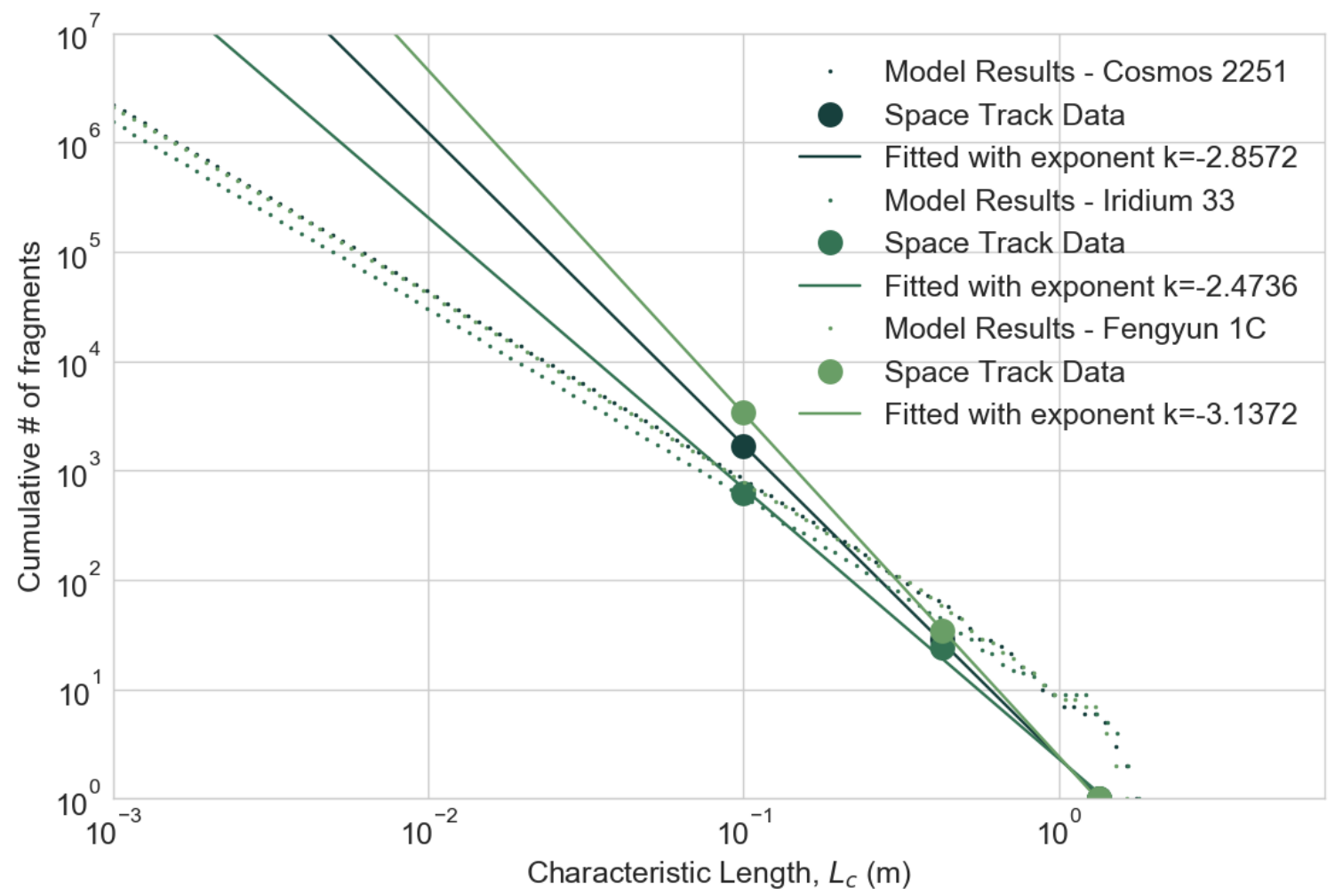

Fig. 11: Comparison of fragment size distribution of observed data against model results for spacecraft collisions.

Fitting trend lines to these data points gives values for the power law of -2.8572, -2.4736 and -3.1372 respectively for Cosmos-2251, Iridium-33 and Fengyun-1C compared to the value of -1.71 used in the model. The average of these three values gives a gradient of -2.8227 which, if used in the model, would provide a significantly different size distribution of fragments. Using the example of the breakup of a $1,000 \mathrm{~kg}$ spacecraft the model currently generates 46,773 fragments greater than $1 \mathrm{~cm}$ in length, however using the averaged value above as the exponent the model would generate 7,859,591, a 168-fold increase. This suggests that the current model may be underestimating the number of fragments in the 1 to $10 \mathrm{~cm}$ range for simulated collisions with significant impacts for the long term evolution of the debris population. 


\section{Impact on Simulations of the Environment}

The impact of the NASA Standard Breakup Model on the results of simulations of the debris environment was noted in Belstead Research Ltd.'s report on debris modelling [24]. A review of published simulation results by year, for the population of objects greater than $10 \mathrm{~cm}$ in size, identified an increase in predicted population following the introduction of the NASA standard Breakup Model. These results highlight the sensitivity of environmental models to the choice of fragmentation model.

The results of the investigation into the output of the NASA Standard Breakup Model highlighted issues which will impact the simulation of the future debris environment. Good agreement with observed results was seen for the size distribution of the breakup of large upper stages which have traditionally been the dominant source of new fragments. However, current trends indicate that a wider range of events will affect the future debris environment such as smaller upper-stages, battery based explosions and, in particular, collisions between objects. The comparison of observed fragmentation debris against the results of the model for examples of these scenarios showed a consistent over-prediction of large fragments above $50 \mathrm{~cm}$ in length and suggest an under-prediction of small fragments.

Within the current design of the model the total original mass is conserved through the inclusion of additional large fragments. However, limitations on the tracking of the smallest fragments gives greater uncertainty to number of smaller fragments indicating that discrepancies in total mass are more likely than with larger well tracked objects.

One consequence of this is that an environment where collisions become the dominant source of new debris may be substantially different to that predicted by current simulations. These results would indicate a shift towards fewer large fragments and many more small fragments with subsequent impacts on evolution of the environment. This would represent a change in the risk profile of the environment.

While this shift may represent more overall fragments being generated in the initial event it is potentially better for the sustainability of the long term environment. Fewer large fragments will result in fewer high mass collisions and so fewer secondary debris fragments being generated in future. At the same time smaller objects tend to have a higher AMR and so be shorter lived due to the increased effect of aerodynamic drag.

However, $1 \mathrm{~cm}$ objects can still be fatal in the event of a collision and the presence of more and harder to track objects is likely to result in a greater rate of collisions. This would represent a significant impact to the ongoing safety of the space environment. The expectation would be of an increased risk to individual spacecraft missions and a higher number of anomalous events and disruption to spacecraft services.

An additional area for consideration is that if or when tracking of $1 \mathrm{~cm}$ objects becomes possible, for instance with the introduction of the Space Fence, there may be many more objects to handle than expected. This will result in much greater overhead for spacecraft operators and their regulators in terms of collision prediction and the management of collision avoidance manoeuvres as well as impacting on future spacecraft design and mission planning.

\section{Conclusions}

Since 1980 there has been clear growth in the number of spacecraft being operated by commercial organisations and these spacecraft now represent a majority of the spacecraft being launched. Accompanying changes can be seen for both the average mass and cross section of spacecraft, two key characteristics used in debris modelling, with noticeable decreasing trends seen in LEO and opposing increasing trends in GEO.

These characteristics are deviating from the assumptions made in many state-of-the-art debris models. In particular, the NASA Standard Breakup Model, a common component of many debris models, is based on empirical evidence gathered from observations of historic spacecraft which are not representative of the modern population.

A comparison of the model against observed fragmentation events indicated good agreement for the traditionally common explosion of large upper-stages. However, the model had a tendency to over-predict the number of larger debris fragments and under-predict the number of small debris fragments when modelling breakups representing the expected future environment.

Previous studies indicate that the choice of fragmentation model has a noticeable impact on the results of simulations. As a result, it is expected that these changes may indicate a meaningful difference between the future environment and the results of current models consisting of more low mass collisions with less debris generation. Overall this represents a priority shift from long term space sustainability towards issues of space safety.

\section{Acknowledgements}

The authors acknowledge support from the University of Southampton EPSRC Centre for Doctoral Training in Next Generation Computational Modelling (Grant No. EP/L015382/1). The authors would also like to thank ESA for providing downloads of spacecraft data from the DISCOS database. 


\section{References}

[1] European Space Agency (ESA), Space debris by the numbers, January 2019, https://www.esa.int/Our_Activities/Operations/Space_Debris/Space_debris_by the_numbers, (accessed 23.09.19).

[2] D. J. Kessler, B. G. Cour-Palais, Collision Frequency of Artificial Satellites: The Creation of a Debris Belt, Journal of Geophysical Research, 83(A6), 2637- 2646.

[3] D. J. Kessler, P. D. Anz-Meador, Critical number of spacecraft in low Earth orbit: using satellite fragmentation data to evaluate the stability of the orbital debris environment, Proceedings of the Third European Conference on Space Debris, 2001, 19 - 21 March.

[4] H. G. Lewis, G. Swinerd, N. Williams, G. Gittins, DAMAGE: a dedicated GEO debris model framework, Proceedings of the Third European Conference on Space Debris, Darmstadt, Germany, 2001, 19 - 21 March.

[5] J. C. Liou, D. T. Hall, P. H. Krisko, J. N. Opiela, LEGEND - A three-dimensional LEO-to-GEO debris evolutionary model, Advances in Space Research, 34(5), (2004) 981-986.

[6] T. Hanada, Orbital debris modeling and applications at Kyushu University. In Procedia Engineering (Vol. 67, pp. 404-411), (2019). Elsevier Ltd. https://doi.org/10.1016/j.proeng.2013.12.040

[7] J. Dolado-Perez, R. Di Costanzo, R. Bruno, Introducing MEDEE- A New Orbital Debris Evolutionary Model, Proceedings of the 6th European Conference on Space Debris. Darmstadt, Germany, 2013, 22 - 25 April.

[8] B. B. Virgili, DELTA (Debris Environment Long Term Analysis), Proceedings of the 6th International Conference on Astrodynamics Tools and Techniques (ICATT), Darmstadt, Germany, 2016, 14 - 17 March.

[9] J. Radtke, S. Mueller, V. Schaus, E. Stoll, LUCA2 - An enhanced long-term utility for collision analysis, Proceedings of the 7th European Conference on Space Debris, Darmstadt. Germany, 2017, 18 - 21 April.

[10] X. Wang, J. Liu, An Introduction to a New Space Debris Evolution Model: SOLEM. Advances in Astronomy, (2019), 2738276. https://doi.org/10.1155/2019/2738276

[11] M. Kerolle. NewSpace - Is this the Advent of the Second Space Age? 23 November 2015, https://www.spaceboard.eu/articles/space-out/newspace-is-this-the-advent-of-the-second-space-age- (accessed 23.09.19).

[12]D. Paikowsky, What Is New Space? The Changing Ecosystem of Global Space Activity. New Space, 5(2), (2017) 84-88.

[13] Bryce Space and Technology, Start-Up Space: Update on Investment in Commercial Space Ventures 2018, https://www.brycetech.com/downloads/Bryce_Start_Up_Space_2018.pdf (accessed 18.01.2019).

[14] J. Radtke, E. Stoll, H. Lewis, B. B. Virgili, The impact of the increase in small satellite launch traffic on the long-term evolution of the space debris environment, Proceedings of the 7th European Conference on Space Debris, Darmstadt. Germany, 2017, 18 - 21 April.

[15] J. Becedas, A. Caparrós, Additive Manufacturing Applied to the Design of Small Satellite Structure for Space Debris Reduction, in: Applications of Design for Manufacturing and Assembly, Ancuţa Păcurar, IntechOpen, 2018.

[16] G. P. Sudhakar, ISRO: 104 Satellites in 1 go, Vidyaniketan Journal of Management Research, Proceedings of International Conference on Strategy, System and Services for Sustainability and Scalability of Business (5S), School of Management, Pondicherry University, Pondicherry, India, 2018, March 23-24.

[17] G. Denis, D. Alary, X. Pasco, N. Pisot, D. Texier, S. Toulza, From new space to big space: How commercial space dream is becoming a reality. Acta Astronautica, (2020), 166, 431-443. https://doi.org/10.1016/j.actaastro.2019.08.031

[18] T. J. Muelhaupt, M. E. Sorge, J. Morin, R. S. Wilson, Space traffic management in the new space era. Journal of Space Safety Engineering, (2019), 6(2), 80-87. https://doi.org/10.1016/j.jsse.2019.05.007

[19] European Space Agency (ESA), DISCOS database (accessed 23.01.2019).

[20]C. Henry, SpaceX plans 24 Starlink launches next year, September 10 2019, https://spacenews.com/spacexplans-24-starlink-launches-next-year/ (accessed 24-09-19).

[21] N. L. Johnson, P. H. Krisko, J. C. Liou, P. D. Anz-Meador, NASA's new breakup model of EVOLVE 4.0, Advances in Space Research, 28(9), (2001), 1377-1384.

[22] J. C. Liou. Collision activities in the future orbital debris environment, Advances in Space Research, 38(9), (2006), 2102-2106.

[23]F. R. Hoots, R. L. Roehrich, Spacetrack Report No. 3--Models for Propagation of NORAD Elements Sets, Spacetrack Report, 3(3), (1980) 1-91. 
[24]J. Beck, Review of Space Debris Population Prediction Methodologies and Results, Belstead Research Ltd., Waterlooville, UK, Rep. no. PR00003/D02, November 2013.

[25] A. B. Jenkin, M. Sorge, G. Peterson, J. McVey, B. Yoo, Recent Enhancements to ADEPT and Sample Debris Environment Projections, IAC-15-A6.2.2, 66th International Astronautical Congress, Jerusalem, Israel, 2015, October 12-16.

[26] P. D. Anz-Meador, J. N. Opiela, D. Shoots, J. C. Liou, History of On-Orbit Satellite Fragmentations, 15th Edition, Orbital Debris Program Office, NASA, Houston, Texas, NASA/TM-2018-220037, July 2018. 